\section{Unterversorgung bereits vorprogrammiert?}

\author{
Nimmt die Politik noch Rücksicht auf allergische Patienten und \\ allergologisch tätige Ärzte? „Allergologie im Brennpunkt der \\ aktuellen Gesundheitspolitik" lautete das brisante Thema eines \\ berufspolitischen Workshops am 5. Februar 2005 im Rahmen des \\ vom ÄDA mit ausgerichteten Allergie-Symposiums München.
}

D erzeit blase den KVen und Vertragsärzten ein rauer Wind ins Gesicht, erklärte Dr. Winfried Goertzen, HNOArzt und Allergologe aus Höchstadt. Forderungen nach "weniger Staat" und „Bürokratieabbau“ oder Kritik am „Widerstand der Ärzte und insbesondere der KVen“" gegen Reformpläne werden zunehmend unverblümter formuliert. Dazu kommt noch die Realitätsferne von Gesundheitsexperten: „Der Betrieb einer Arztpraxis ist praktisch risikolos" (Karl Lauterbach, Köln).

\section{Patientenschwund dank Praxisgebühr}

Die Einführung der Praxisgebühr führte dazu, dass viele Patienten ihre Ärzte nicht mehr aufsuchen. Gerade allergologisch tätige Ärzte wie Dermatologen und HNO-Ärzte verzeichneten einen extremen Rückgang der Fallzahlen: Im Vergleich der ersten Quartale der Jahre 2003 und 2004 verringerte sich beispielsweise in Bayern bei den Dermatologen die Fallzal um 17,2\%, bei den HNO-Ärzten um $13,2 \%$. Auch im Vergleich der zweiten Quartale war noch eine deutliche

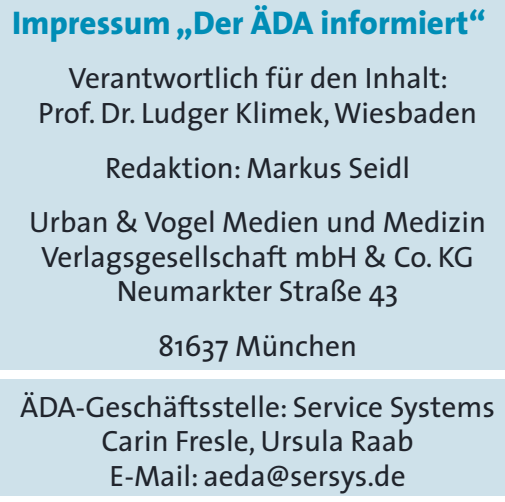

Reduktion zu verzeichnen, bei dem Dermatologen um $14,9 \%$, bei den $\mathrm{HNO}$ Ärzten um 9,2\%

Goertzen betonte, dass der Steuerungseffekt der Praxisgebühr nicht zwischen nützlichen oder überflüssigen Leistungen differenziere. Zudem seien es häufig sozial Schwache, die von einem Arztbesuch Abstand nehmen.

\section{Allergie - nur eine Bagatell- erkrankung?}

Die Verordnung von rezeptfreien Antihistaminika zu Lasten der gesetzlichen Krankenkassen ist nur noch in seltenen Ausnahmen wie für Notfallsets für Insektenstiche, schwere rezidivierende Urtikaria oder schwerwiegenden anhaltenden Pruritus möglich. Rezeptpflicht besteht nur noch für wenige Antihistaminika, deren Patentschutz noch nicht abgelaufen ist. Damit stufte die Gesundheitspolitik Allergien in den Augen vieler als Bagatellerkrankungen ein. Den Patienten vermehrt rezeptpflichtige Antihistaminika zu verschreiben, ist den Vertragsärzten verwehrt: Bei verändertem Verordnungsverhalten droht u. U. ein Regress. Goertzen empfahl für die Verordnung von rezeptpflichtigen Antihistaminika eine exakte und ausführliche Dokumentation.

Gemäß dem „Weißbuch Allergologie in Deutschland" sind nur $10 \%$ der Patienten mit Allergien entsprechend den gültigen Empfehlungen der Fachverbände therapiert. „Allergologische Patientenkarrieren sind schlechte Karrieren!", warnte Goertzen. Eine Befragung bei knapp 6.800 Betroffenen zeigte, dass oft erst eine längere frustrane allergologische Vorgeschichte zum Spezialisten führte. $51,3 \%$ suchten ihn auf eigene Initiative auf, nur 29,8\% wurden überwiesen. $31,8 \%$ der Patienten befanden sich in Behandlung bei Nicht-Allergologen (Allergo J 2003; 12: 521-6). Insbesondere der Zugang zur spezifischen Immuntherapie ist für viele Patienten erschwert. Die Schnittstelle zwischen Allergologen und Nicht-Allergologen funktioniert nur begrenzt.

In Bayern ist ein Strukturvertrag geplant, der die Versorgung allergologischer Patienten verbessern soll. Hier soll die kompetente Behandlung im Vordergrund stehen. Schulungsprogramme und Einhaltung hoher Qualitätsstandards sind ebenso Voraussetzung für die Teilnahme wie die effiziente Zusammenarbeit zwischen Hausarzt und Facharzt.

\section{Wozu noch „den Allergologen“?}

Allergologische diagnostische und therapeutische Leistungen sind keineswegs mehr den Allergologen vorbehalten. Im neuen EBM 2000 plus ist die allergologische Basisdiagnostik für Hausärzte, Kinderärzte und Internisten mit oder ohne Fachschwerpunkt mit jeweils 220 Punkten einmal pro Quartal möglich. Fachinternisten können die allergologische Basisdiagnostik auch im Rahmen des fachinternistischen Basiskomplexes (420 Punkte) abrechnen.

Der allergologisch-diagnostische Komplex für eine (Kontakt-)Allergie vom Typ IV (Nr. 30110; 1.720 Punkte) oder für eine Allergie vom Typ I (Nr. 30111; 1.245 Punkte) ist nicht nur für Ärzte mit der Zusatzbezeichnung Allergologie, sondern auch für HNO-Ärzte, Dermatologen, Internisten mit dem Schwerpunkt Pneumologie und Kinderärzte - jeweils ohne Zusatzbezeichnung - möglich. Die Nr. 30111 ist nur einmal im Jahr abrechnungsfähig. Spätestens ab Januar 2006 sollen bedarfsorientierte Regelleistungsvolumina gelten, spezielle Zusatzbudgets für die Allergologie sind dann nicht mehr vorgesehen.

Goertzen appellierte an die Zuhörer als Ärzte mehr Lobbyarbeit zu betreiben und auf allen Ebenen Druck auf die Politiker auszuüben. Beim geplanten Präventionsgesetz sollte die Allergologie mit berücksichtigt werden. Die Versorgung allergologischer Patienten „darf nicht zum Spielball einer auf kurze Erfolge orientierten Politik werden“. af 